\title{
Image Watermarking Using All Phase Discrete Cosine Biorthogonal Transform in Selected Pixel Blocks
}

\author{
Farhad M. Khalifa ${ }^{1 *}$, Mohammed G. Saeed ${ }^{2}$ \\ ${ }^{1}$ Department of Computer Networks, Bardarash Technical Institute, Duhok Polytechnic University, Duhok, Kurdistan Region, Iraq, \\ ${ }^{2}$ Department of Information Technology, Shekhan Technical Institute, Duhok Polytechnic University, Duhok, Kurdistan Region, Iraq.
}

\author{
${ }^{*}$ Corresponding author: \\ Farhad M. Khalifa, \\ Department of Computer \\ Networks, Bardarash \\ Technical Institute, Duhok \\ Polytechnic University, \\ Duhok, Kurdistan Region, \\ Iraq. \\ E-mail: farhad.khalifa@dpu. \\ edu.krd
}

Received: 10 September 2019

Accepted: 17 June 2020

Published: 30 June 2020

\section{DOI}

10.25156/pti.v10n1y2020.pp68-73

\begin{abstract}
A B S TR A C T
In the past decade, a transform called all phase discrete cosine biorthogonal transform (APDCBT) appeared in the field of digital image processing. It is mainly used to solve the negativity found in discrete cosine transform (DCT), especially in a low bit rate. In this paper, the APDCBT is employed for watermark insertion based on selected regions of an image. The insertion is depending on the homogeneity of each part of the image. To determine the extent of homogeneity, there are two criteria: Mean and standard deviation are applied for the intensity of the image. Medium frequency bands of APDCBT in image pixel blocks are used to hold the embedded watermark. Then, the transform is inversed to obtain the resultant watermarked image. The robustness of APDCBT against watermark removal attacks is tested and the experimental results showed the superiority of the APDCBT over traditional DCT in the watermark embedding system. More specifically, when the LSB reset attack applied. For instance, when the contrast adjustment attack applied, the average of normalized crosscorrelation (NCC) values of extracted watermark images with the original watermark, it was 0.992 for the proposed method. This is a promised result, if it is compared with the NCC of the DCT method, which was 0.423 . The proposed method can be used for copyright protection purpose.
\end{abstract}

Keywords: All phase discrete cosine biorthogonal transform; Discrete cosine transform; Image watermark; Information hiding; Selected pixel blocks

\section{INTRODUCTION}

Multimedia information, including image, has become one of the most communication contents. Due to the fast growth of internet and boundless use of image processing and multimedia technologies, People can easily obtain the extensive multimedia files through the internet, create problems such as prohibited copying, forge ownership claims, because of the ease of copying or altering digital documents quickly and without quality loss (Saboori and Hosseini, 2014). The watermarking system has been proposed for avoiding these problems (Hasoon and Khalifa, 2012; Hosseini and Saboori, 2015).

There are two groups of techniques to add watermark to images according to their domain. The first group is the spatial domain-based watermarking methods, in which the watermarking information are inserted into carrier image by adjusting its pixel values, although the spatial domain techniques need less computation power and have less execution time, however, they do not have the ability to resist versus various attacks, the second group is transform domain-based watermarking methods (Hosseini and Saboori, 2015; Zhou et al., 2018).
Transform domain is more robust than spatial domain methods. In techniques that use the transform domain, the carrier image converted into the frequency domain. Before embedding a robust watermark into host image, researchers commonly tune the coefficients of a transform such as DFT, DCT, or DW'T (Yantao et al., 2008; Apostol et al., 2013).

In Zhou et al., 2018, the discrete wavelet transform (DWT) and all phase discrete cosine biorthogonal transform (APDCBT) presented with singular value decomposition (SVD). To enhance the watermark imperceptibility, the authors concluded that the proposed scheme has little detectable effect on the original image, and it shows better robustness against typical signal processing attacks than other algorithms.

In Abraham and Paul, 2014, algorithm was proposed to ensure higher visual quality after watermark addition to image, blocks or pixel regions selected for watermark are hiding such as regions in the image. Where modifications are least noticeable by human eyes, the outcome of proposed was successful in surviving even after four bits on the least significant side of all pixels in the image was reset. 
In Khalifa et al., 2019, a hybrid technique of DWT and DCT used to provide advantages of both techniques. The DWT has the disadvantage of fraction loss in watermark insertion which rises mean square error (MSE) and the results decreasing peak signal to noise ratio (PSNR) where PSNR of the recovered secret image is higher compared to existing DWT and DCT techniques.

\section{APDCBT and DCT}

It is known, for researches of the discrete cosine transformation (DCT) field, that the DCT has good properties related to energy concentration. Therefore, it has been employed in many applications, such as image compression, watermarking, and object recognition (Agrwal et al., 2016; Zhou et al., 2018). This DCT technique is used to convert images or signals from space time into the spectral domain. This conversion has the power to show perfect energy compaction. The transformation between these domains is linear, which save the energy of the real coefficients, to become as a good frequency features of the space and time (Kannan and Gobi, 2015). It also shared that dividing the low, high, and average frequencies from each other, will influence the chance of using the coefficients with the high frequencies group rather than others. In general, the DCT applied on Windows (blocks) of $8 \times 8$ pixels instead of using the whole image (Mokhnache et al., 2018). However, there are some negative properties, which not covered when the image is compressed using the DCT with a Window that has low bit rates. To overcome this issue, the APDCBT transform is applied since if it compared with the conventional DCT, this transform (APDCBT) is offering good performance in both high and low frequencies (Wang et al., 2013). Because of this performance, the APDCBT has been widely used in the image processing in general and in the watermarking in special. In this paper, the APDCBT transform employed as a watermarking style, where this transform has a transformation matrix, which is called $\mathrm{B}$, with a dimension of $N \times N$, as defined in Equation (1) (Manogna and Chaitanya, 2018).

$$
B(m, n)=\left\{\begin{array}{c}
\frac{N-m}{N^{2}}, \quad m=0,1, \ldots, N-1, n=0 \\
\frac{1}{N^{2}}\left[(N-m) \cos \frac{m n \pi}{N}-\csc \frac{n \pi}{N} \sin \frac{m n \pi}{N}\right], \\
m=0,1, \ldots, N-1, n=1,2, \ldots, N-1
\end{array}\right.
$$

For an image block $X$ with a dimension of $N \times N$, the APDCBT can be expressed as follows:

$$
Y=B X B^{T}
$$

Where, $Y$ denotes the transform coefficient matrix after APDCBT.

\section{METHODOLOGY}

Watermarking includes two rounds of operations, an embedding and an extraction. There are two important processes in the embedding operation phase: Selecting the regions or blocks of image pixels and domain transforming.

Enforcing the watermark is achieved through the embedding process after transforming the original image from the spatial to the frequency domain using APDCBT block wise.

APDCBT transform decomposes an image into three frequency bands, namely, low, medium, and high frequencies, (LF), (MF), and (HF) regions, respectively (Abraham and Paul, 2014). Figure 1 illustrates the three distinct areas.

MF bands are more suitable for watermark embedding where adjustments do not affect image quality negatively. The watermark signal is added to the MF zone coefficients in selected blocks to ensure being difficult to perceive.

\section{Blocks Selection for Watermarking}

This work is based on hiding data in selected image pixel blocks depending on the homogeneity of the image area. Specific criteria were used to examine the homogeneity of these areas of the image. The average of the image pixels intensity calculated and compared with the standard deviation of those pixels. A heterogeneous image area forms a convenient environment for hiding data without the appearance of concealment because hiding in plain areas makes the embedded data exposed and distort the cover image.

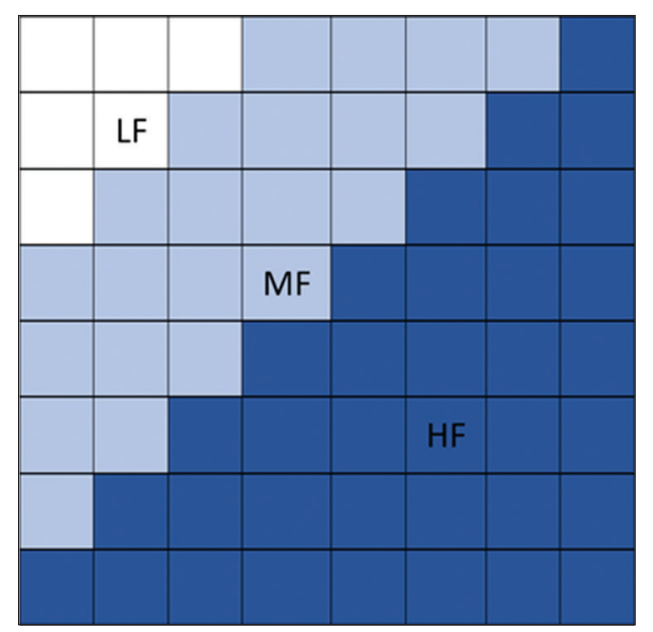

Figure 1: All phase discrete cosine biorthogonal transform three distinct areas according to frequency bands 
In a homogeneous image area, the region is almost monotonous. Any modifications to these areas are distinguishable. However, heterogeneous image region, pixels vary by larger intensity values. Hence, alteration on to these areas will be least distracting to the human visual system.

Non-overlapped image pixel blocks of size $8 \times 8$ adopted in this paper. Before embedding the data, these blocks tested by finding the average of pixels intensity using Equation (1), and the standard deviation using Equation (2), then comparing them using a threshold. The threshold is a fixed number empirically determined. Increasing threshold value leads to more imperceptibly in the watermark.

\section{Watermark Embedding}

Watermark insertion operation illustrated in Figure 2. IW is the watermarked image, W is the watermark, and I is the original image. Once image pixel block selected, it transformed using APDCBT resulting in an $8 \times 8$ matrix, which is divided into three bands as previously mentioned, $\mathrm{LF}, \mathrm{MF}$, and HF. The only medium frequency band is used to hold the watermark. If $w m(t)==0, f w(i, j)=s f \times f(i, j)$; where $w m(t)$ is a watermark bit to be added and $t=1,2$ ...L, where $L$ is the length of the watermark, $f(i, j)$ is a medium frequency APDCBT coefficient from the block, $s f$ is a modification factor, and $f w$ is the modified element. However, if $w m(t)==1$, leave the coefficient unaffected. Finally, compute inverse APDCBT of the block resulting in the watermarked image. As Figure 1 clarify, MF band forms 22 pixels out of 64 pixels in each block, that is, every selected block of the cover image can hold 22 bits of the watermark.

\section{Algorithm 1: Embedding process}

1. Input the image and decompose it into blocks of size $(8 \times 8)$.

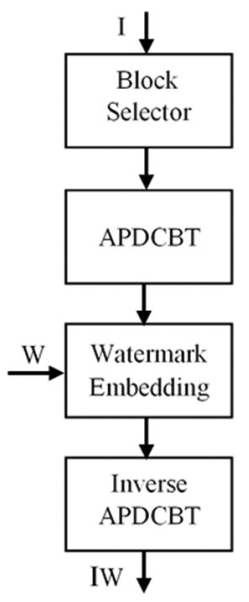

Figure 2: Proposed embedding method
2. Compute the block average (av) and standard deviation $(s d)$ of the block's intensity value.

3. If $\left(s d>t b^{*} a v\right)$ is not true, go to previous step for computations on next block. Otherwise, select the block for watermark embedding and go to next step.

4. Compute APDCBT of the block.

5. For all mid-frequency APDCBT coefficient element from the selected block, if the correspond watermark bit is zero modify the element by some factor otherwise leave it.

6. Compute inverse APDCBT of the block.

7. If there are more blocks left, go to step 2 for computations on next block.

8. Output the watermarked image.

\section{Watermark Extraction}

Figure 3 illustrates the operation of watermark extraction. The importance of the hidden watermark extraction process is not less than the importance of the embedding. Only successful recovery of the watermark can establish resource ownership. The process of watermark extraction takes the original clean image and the watermarked image. Moreover, outputs hidden watermark.

The original clean image is used to select the same selected blocks in the embedding phase. Using the original image for block selecting is critical because the changing in watermarked image pixel values due to the embedding process can affect the results. After block selected in the same way as the embedding phase, it transformed using APDCBT in parallel with computing the APDCBT of the identical block in the watermarked image, then compare the APDCBT coefficients of both blocks from a similar position. if $f w(i, j)>f(i, j), w m(t)==0$ otherwise $w m(t)==1$; where $f$ and $f w$ are medium frequency APDCBT coefficients from a similar position from the blocks in original clean image and watermarked image. Moreover, wm is the

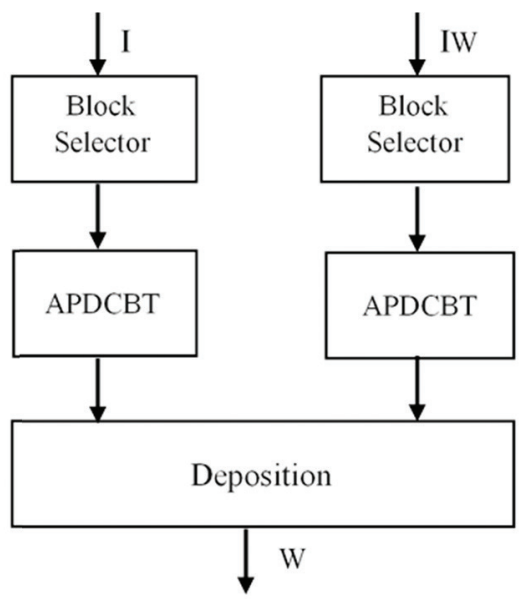

Figure 3: Proposed extraction method 
watermark bit stream. This process continues until the watermark image is retrieved.

Algorithm 2: Extraction process

1. Input the original image and watermarked image, then decompose them into blocks of size $8 \times 8$.

2. Compute the block average (av) and standard deviation $(s d)$ of the block's intensity value of the original image.

3. If $\left(s d>t b^{*} a v\right)$ is not true, go to previous step for computations on next block. Otherwise, select the block for watermark extraction and go to next step.

4. Compute APDCBT of the block of original image and correspond block from watermarked image.

5. For all mid-frequency APDCBT coefficient elements from the selected block, compare similar position elements from original image and watermarked image, if the element of watermarked is greater than original, then correspond watermark bit is zero. Otherwise, watermark bit is one.

6. If there are more blocks left, go to step 2 for computations on next block.

7. Output the watermark image.

\section{EXPERIMENTAL RESULTS ANALYSIS}

Use the proposed algorithm tested on eight standard 512 $\times 512$ grayscale images, Barbara, Boats, Bridge, Couple, Fingerprint, Hill, Lake, and Lena. A $32 \times 32$ binary image used as a watermark. All used images and the watermark, as shown in Figure 4.

MSE is called the square of differences in the pixel values between the corresponding pixels of the two images. The MSE of $N \times M$ size image is given by Equation (3) (Mohammad et al., 2017). PSNR is a geometric name, for the ratio between the maximum possible power of a signal and the power of vitiation noise that affects the accuracy (Brifcani and Al-Bamerny, 2010; Yamini, 2018).

MSE (3) and PSNR (4) analysis shown in Table 1. For good watermarking, low values of PSNR are wondered. On the other hand, low values of MSE means imperceptible watermark. Boat image shows the best PSNR and MSE results, whereas fingerprint image has the worst ones.

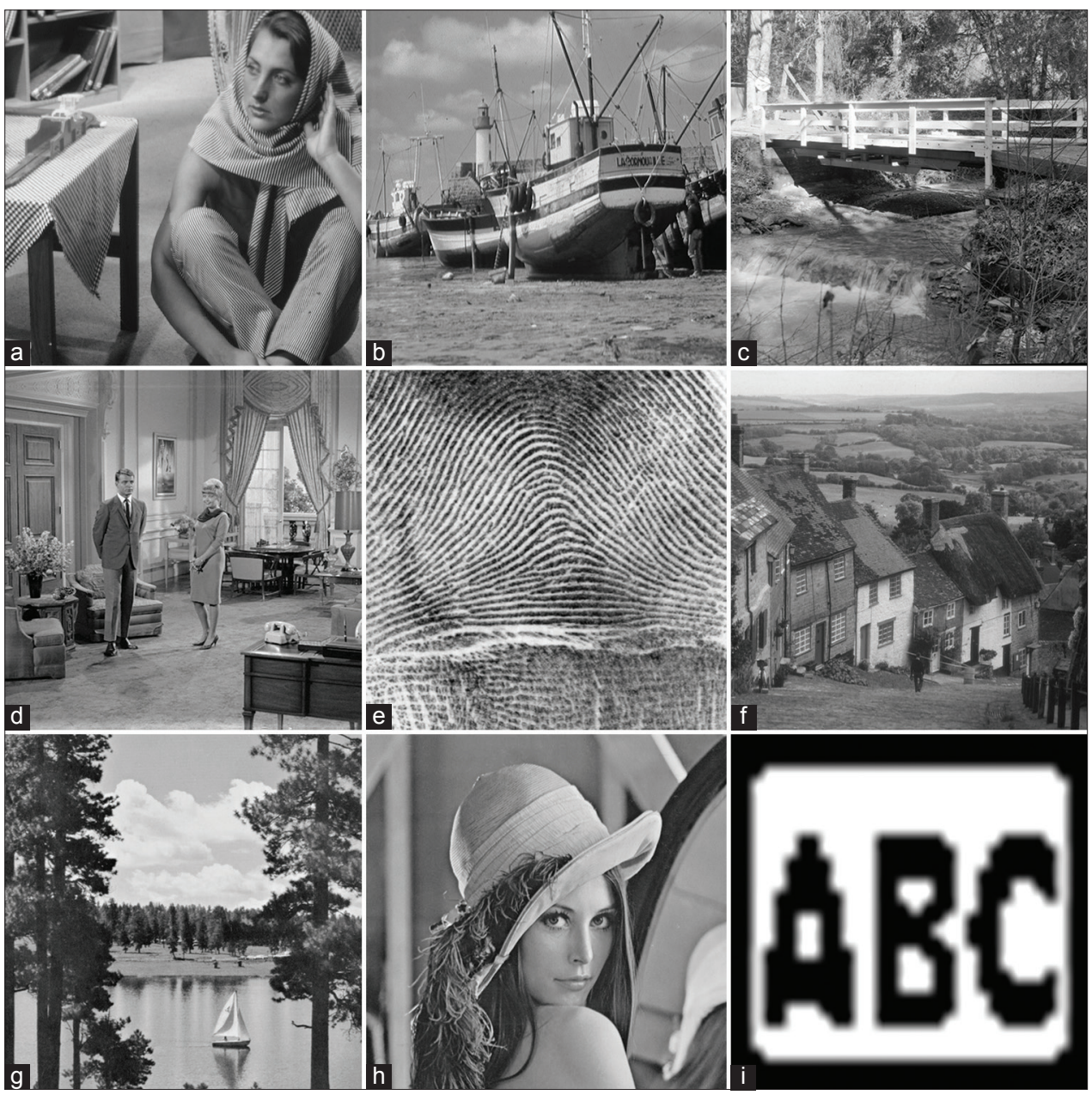

Figure 4: Original cover images and watermark. (a) Barbara, (b) Boats, (c) Bridge, (d) Couple, (e) Fingerprint, (f) Hill, (g) Lake, (h) Lena, (i) $\mathrm{ABC}$ watermark 


$$
\begin{gathered}
M S E=\frac{1}{m n} \sum_{i-0}^{m-1} \sum_{j=0}^{n-1}[I(i, j)-K(i, j)]^{2} \\
\text { PSNR }=10 . \operatorname{LOG}_{10} \frac{M A X_{I}^{2}}{M S E}
\end{gathered}
$$

The proposed method tested under a watermark removal attack with four levels of LSB reset attack to checking its robustness against attacks. This kind of attack resets a number of bits from the right side, that is, from the LSB side. In this work, $8^{\text {th }}, 7^{\text {th }}-8^{\text {th }}, 6^{\text {th }}-8^{\text {th }}$, and $5^{\text {th }}-8^{\text {th }}$ LSB reset to zero each one in separated operation. Other attacks, JPEG compression, Gaussian noise, histogram equalization, and contrast adjustment, also applied.

To compare the performance of APDCBT used in the proposed method, the DCT method proposed in Abraham and Paul, 2014 tested under the same attacks. The normalized cross-correlation (NCC) values for extracted watermark images with the original watermark are calculated using both methods. Table 2 shows the average of NCC values for the APDCBT proposed method and the DCT method with LSB reset attack, whereas Table 3 shows the results with other attacks.

The experimental results, Table 2 and Figure 5, proved the robustness of APDCBT against LSB resetting attack in comparison to DCT in the watermark embedding system. However, testing both methods under JPEG

Table 1: Experimental results

\begin{tabular}{lcc}
\hline Images & MSE & PSNR \\
\hline Barbara & $8.35 \mathrm{E}-06$ & 50.4728459 \\
Boats & $1.28 \mathrm{E}-05$ & 48.9276355 \\
Bridge & $1.06 \mathrm{E}-05$ & 49.7361311 \\
Couple & $1.07 \mathrm{E}-05$ & 49.7085744 \\
Fingerprint & $6.28 \mathrm{E}-06$ & 52.0171729 \\
Hill & $7.96 \mathrm{E}-06$ & 50.3163369 \\
Lake & $7.29 \mathrm{E}-06$ & 50.811477 \\
Lena & $1.00 \mathrm{E}-05$ & 49.9880453 \\
\hline
\end{tabular}

compression, Gaussian noise, histogram equalization, and contrast adjustment attacks, Table 3 and Figure 6, showed

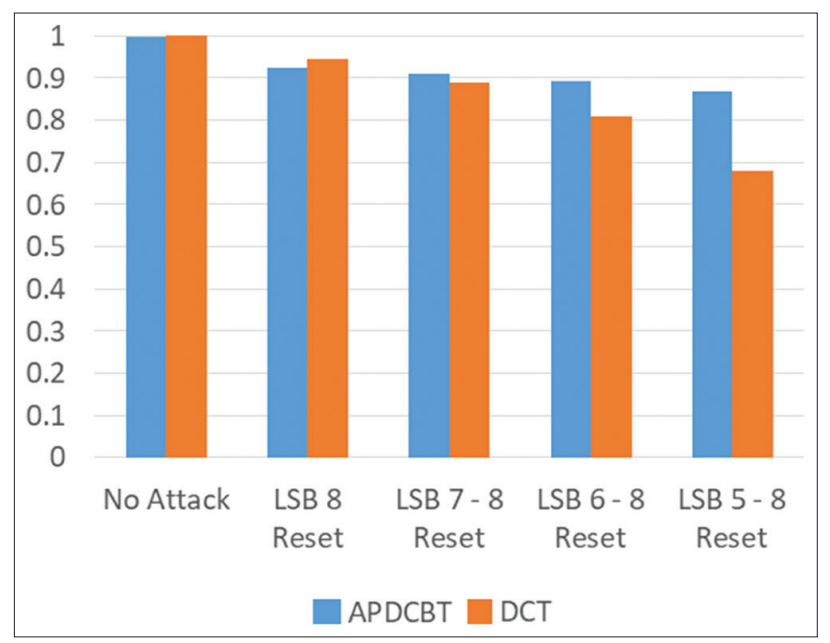

Figure 5: Comparison of average normalized cross-correlation of all images with LSB reset attack for the proposed method and DCT method

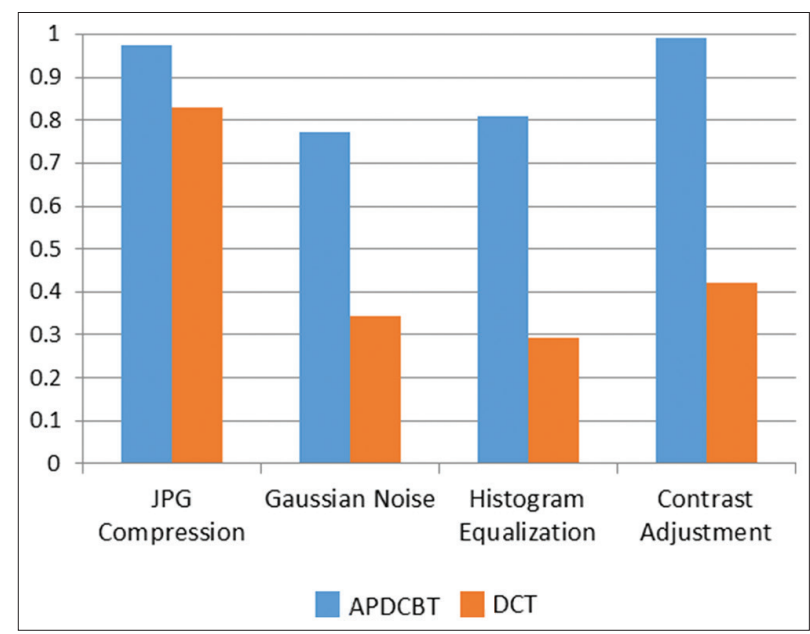

Figure 6: Comparison of average normalized cross-correlation of all images under JPEG compression, Gaussian noise, histogram equalization, and contrast adjustment attacks for the proposed method and DCT method

\begin{tabular}{|c|c|c|c|c|c|c|c|c|c|c|}
\hline \multirow[t]{2}{*}{ Images } & \multicolumn{2}{|c|}{ No attack } & \multicolumn{2}{|c|}{ LSB 8 reset } & \multicolumn{2}{|c|}{ LSB 7-8 reset } & \multicolumn{2}{|c|}{ LSB 6-8 reset } & \multicolumn{2}{|c|}{ LSB 5-8 reset } \\
\hline & APDCBT & DCT & APDCBT & DCT & APDCBT & DCT & APDCBT & DCT & APDCBT & DCT \\
\hline Barbara & 1 & 1 & 0.9 & 0.91 & 0.904 & 0.818 & 0.889 & 0.735 & 0.882 & 0.579 \\
\hline Boats & 0.998 & 1 & 0.964 & 0.947 & 0.915 & 0.91 & 0.889 & 0.825 & 0.868 & 0.714 \\
\hline Bridge & 1 & 1 & 0.987 & 0.987 & 0.996 & 0.989 & 0.887 & 0.934 & 0.885 & 0.821 \\
\hline Couple & 1 & 1 & 0.944 & 0.944 & 0.927 & 0.87 & 0.893 & 0.793 & 0.885 & 0.622 \\
\hline Fingerprint & 0.998 & 1 & 0.895 & 0.974 & 0.887 & 0.934 & 0.88 & 0.859 & 0.857 & 0.761 \\
\hline Hill & 1 & 1 & 0.923 & 0.929 & 0.887 & 0.842 & 0.917 & 0.712 & 0.887 & 0.615 \\
\hline Lake & 1 & 1 & 0.904 & 0.944 & 0.891 & 0.897 & 0.882 & 0.842 & 0.84 & 0.726 \\
\hline Lena & 0.998 & 1 & 0.87 & 0.936 & 0.874 & 0.844 & 0.9 & 0.769 & 0.855 & 0.6 \\
\hline Average & 0.999 & 1 & 0.923 & 0.946 & 0.91 & 0.888 & 0.892 & 0.809 & 0.87 & 0.68 \\
\hline
\end{tabular}

Table 2: Normalized cross-correlation with LSB reset attack 
Table 3: Normalized cross-correlation with (JPEG compression, Gaussian noise, histogram equalization, and contrast adjustment) attacks

\begin{tabular}{|c|c|c|c|c|c|c|c|c|c|c|}
\hline \multirow[t]{2}{*}{ Images } & \multicolumn{2}{|c|}{ No attack } & \multicolumn{2}{|c|}{ JPEG compression } & \multicolumn{2}{|c|}{ Gaussian noise } & \multicolumn{2}{|c|}{ Histogram equalization } & \multicolumn{2}{|c|}{ Contrast adjustment } \\
\hline & APDCBT & DCT & APDCBT & DCT & APDCBT & DCT & APDCBT & DCT & APDCBT & DCT \\
\hline Barbara & 1 & 1 & 0.951 & 0.882 & 0.791 & 0.417 & 0.637 & 0.35 & 1 & 0.979 \\
\hline Boats & 0.998 & 1 & 0.991 & 0.795 & 0.782 & 0.297 & 0.756 & 0.186 & 1 & 0.673 \\
\hline Bridge & 1 & 1 & 0.955 & 0.874 & 0.778 & 0.447 & 0.979 & 0.37 & 0.983 & 0.13 \\
\hline Couple & 1 & 1 & 0.994 & 0.835 & 0.808 & 0.38 & 0.726 & 0.278 & 0.981 & 0.641 \\
\hline Fingerprint & 0.998 & 1 & 0.989 & 0.771 & 0.784 & 0.207 & 0.553 & 0.083 & 1 & 0.444 \\
\hline Hill & 1 & 1 & 0.949 & 0.85 & 0.752 & 0.389 & 0.921 & 0.528 & 0.989 & 0.244 \\
\hline Lake & 1 & 1 & 0.985 & 0.799 & 0.765 & 0.312 & 0.938 & 0.237 & 0.991 & 0.068 \\
\hline Lena & 0.998 & 1 & 0.989 & 0.831 & 0.726 & 0.301 & 0.968 & 0.314 & 0.994 & 0.203 \\
\hline Average & 0.999 & 1 & 0.975 & 0.83 & 0.773 & 0.344 & 0.81 & 0.293 & 0.992 & 0.423 \\
\hline
\end{tabular}

the supreme resistance of the proposed method against those attacks in comparison to DCT method.

\section{CONCLUSION}

Watermark influences the original image as it alters some of the original image information. To minimize the influence of adding watermark and also to protect embedded watermark from attacks, watermark inserted into the safer image regions. Therefore, the embedding is done in selected heterogeneous image pixel blocks.

The present work examines the performance of APDCBT in image watermarking in comparison with using traditional DCT. Furthermore, the robustness of APDCBT against watermark removal attack tested. The experimental results, Figure 5, proved the robustness of APDCBT in comparison to DCT in the watermark embedding system. APDCBT showed better results than DCT by increasing the level of LSB resetting. The watermark successfully survived even after eliminating half of the bits of the watermarked image. PSNR and MSE analysis showed the proposed method as a good watermarking. The size of watermark is depend on the cover image homogeneity since the average of pixels intensity and the standard deviation of any block determine if it can hold the watermark data or not.

\section{REFERENCES}

Abraham, J. and V. Paul. 2014. Image Watermarking Using DCT in Selected Pixel Regions. In: International Conference on Control, Instrumentation, Communication and Computational Technologies. p398-402.

Agrwal, S. L., A. Yadav, U. Kumar and S. K. Gupta. 2016. Improved Invisible Watermarking Technique Using IWT-DCT. In: $5^{\text {th }}$ International Conference on Reliability, Infocom Technologies and Optimization (Trends and Future Directions). p283-285.

Apostol, C. G., D. M. Pîrloaga, M. Rogobete and C. Răcuciu. 2013. Using the Chaos theory and dynamic keys in digital watermarking. Mircea cel Batran Nav. Acad. Sci. Bull. 16:
171-175. Available from: https://www.anmb.ro/buletinstiintific/ eng/2013/ABSTRACT_BulSt_2013_1_FCS.pdf. [Last accessed on 2019 Jan 20].

Brifcani, A. M. A. and J. N. Al-Bamerny. 2010. Image Compression Analysis Using Multistage Vector Quantization Based on Discrete Wavelet Transform. In: International Conference on Methods and Models in Computer Science. p46-53.

Hasoon, S. O. and F. M. Khalifa. 2012. Steganalysis using KL transform and radial basis neural network. AL-Rafidain $\mathrm{J}$. Comput. Sci. Math. 9(1): 47-58.

Hosseini, S. A. and A. Saboori. 2015. A New Method for Color Image Watermarking Based on Combination of DCT and PCA. In: International Conference on Communications, Signal Processing, and their Applications. p1-5.

Kannan, D. and M. Gobi. 2015. An extensive research on robust digital image watermarking techniques: A review. Int. J. Signal Imaging Syst. Eng. 8(1-2): 89-104.

Khalifa, I. A., M. Ataş, S. R. M. Zeebaree, F. Khalifa. 2019. Image steganalysis in frequency domain using co-occurrence matrix and Bpnn. Sci. J. Univ. Zakho. 7(1): 27-32.

Manogna, S. A. and K. Chaitanya. 2018. A color image watermarking scheme based on QR factorization, logistic and lorentz chaotic maps. Int. J. Sci. Dev. Res. 3(7): 334-342.

Mohammad, O. F., M. S. M. Rahim and O. F. Mohammad. 2017. A survey and analysis of the image encryption methods. Int. J. Appl. Eng. Res. 12(23): 13265-13280.

Mokhnache, S., T. Bekkouche and D. Chikouche. 2018. A robust watermarking scheme based on DWT and DCT using image gradient. Int. J. Appl. Eng. Res. 13(4): 1900-1907.

Saboori, A. and S. A. Hosseini. 2014. A New Method for Digital Watermarking Based on Combination of DCT and PCA. In: $22^{\text {nd }}$ Telecommunications Forum Telfor. p521-524.

Wang, C. Y., B. C. Jiang and S. Z. Xie. 2013. Properties of all phase biorthogonal transform matrix and its application in color image compression. J. Comput. Inf. Syst. 9(18): 7227-7234.

Yamini, M. M. A. 2018. Peak signal to noise ratio \&mean square error calculation for various images using the lossless image compression in CCSDS algorithm. Int. J. Pure Appl. Math. 119(12): 14471-14477.

Yantao, Z., M. Yunfei and L. Zhiquan. 2008. A Robust ChaosBased DCT-Domain Watermarking Algorithm. In: International Conference on Computer Science and Software Engineering. p935-938.

Zhou, X., H. Zhang and C. Wang. 2018. A robust image watermarking technique based on DWT, APDCBT, and SVD. Symmetry. 10(3): 77. 